\title{
On the Influence of Electricity Demand Patterns, Battery Storage and PV System Design on PV Self-Consumption and Grid Interaction
}

\author{
Geert Litjens, Wilfried van Sark, Ernst Worrell \\ Copernicus Institute of Sustainable Development, Utrecht University \\ PO Box 80.115, 3508TC Utrecht, the Netherlands
}

\begin{abstract}
New policies supporting photovoltaics (PV) selfconsumption and reduced feed-in power flows are advocated in many countries. Consequently, knowledge about the influences of demand patterns and battery energy storage on PV selfconsumption and grid impact is required. This study analysed these influences for 400 residential and 26 commercial systems from the Netherlands. Results show larger self-consumption rates for commercial systems than residential systems. Batteries increase the self-consumption rate depending on the PV size and the battery size. Averaged curtailment losses are larger for residential systems than commercial systems and decrease with the addition of a battery to the system.

Index Terms-PV self-consumption, battery size, demand patterns, curtailment losses
\end{abstract}

\section{INTRODUCTION}

The increase in deployment of photovoltaic (PV) systems for residential and commercial buildings will result in larger and difficult to predict power and voltage fluctuations, impacting the reliability and power quality of the low voltage grid. Consequently, new policy incentives supporting PV selfconsumption are advocated in many European countries, e.g. feed-in limitation or reducing feed-in tariffs. These policies are expected to open new markets for PV systems designed for local energy demand [1]. PV systems integrated with storage technologies or demand side management result in larger shares of self-consumed PV energy. However, knowledge of demand patterns is required to determine this relationship in more detail. Understanding the potential of self-consumption is essential to calculate the economical profitability of PV systems and batteries. Additionally, batteries could store PV peak production, consequently reducing the grid impact.

A recent review study investigated the increase in selfconsumption rates for PV systems with batteries and found 13 to $24 \%$ points increase with a battery capacity of $0.5-1$ kWh per kWp installed PV capacity. [2]. Another study found that PV systems with batteries will be a more economical solution in the long term than PV systems without battery [3]. Furthermore, applying predictive charge control strategies for PV battery systems contributes to the reduction of PV peak power injection in the grid, and consequently reducing curtailment losses. [4]. Moreover, the curtailment loss is mainly dependent on the battery algorithm used [5].

The aim of this research is to analyse the influence of residential and commercial demand patterns on the annual self-consumption rates and annual curtailment losses. These parameters are studied for different PV system and battery sizes. Most previous research used case studies that included fewer demand patterns, whereas this study includes 400 residential and 26 commercial demand patterns. Consequently, this study presents the self-consumption and curtailment loss values for a range of demand patterns, leading to new insights of the impact of demand patterns, which adds knowledge to the current literature.

\section{Methods}

\section{A. Parameters}

Two major parameters are investigated during this research; Self-Consumption Rate (SCR) and curtailment loss. The annual SCR is defined as the ratio between the annual directly consumed PV energy and the annual total produced PV energy, see Eq.(1).

$$
\text { Self-consumption rate }=\frac{E_{\text {direct consumed }}}{E_{\text {total produced }}}
$$

The second parameter is the curtailment loss, which was calculated according to Eq. (2). The feed-in limitation is specified by $P_{\lim }$ and was set to $0.5 \mathrm{~kW} / \mathrm{kWp}$. This limitation is considered as requirement to obtain grants and loans for PV energy storage systems from the German development bank KfW and also used in previous studies [5], [6].

$$
\begin{array}{r}
P=P_{P V}-P_{\text {demand }}-P_{\text {battery }} \\
P_{\text {grid }}= \begin{cases}P & \text { if } P \leq P_{\text {lim }} \\
P_{\text {lim }} & \text { if } P>P_{\text {lim }}\end{cases} \\
\text { Curtailment loss }=\frac{\sum P-\sum P_{\text {grid }}}{\sum P_{\mathrm{PV}}}
\end{array}
$$

\section{B. Model}

The analysed parameters are modelled using an in-house developed Urban Energy Flow Model (UEFM), written in Python. The aim of this model is to investigate the integration of renewable resources in urban areas from a technological and economical perspective. Within this model time series of a 5 minutes interval are generated and analysed. This model requires energy supply and demand patterns. The energy supply patterns were modelled with the open source package PVLIB [7]. This package provides functions to model the PV yield using atmospheric and PV system parameters. This model uses radiation data with 10 minute interval measured 
by the Royal Dutch Meteorological Institute in de Bilt (The Netherlands). The module parameters of the Sanyo HIP$225 \mathrm{HDE} 1$ and the inverter parameters of the Enphase Energy M250, obtained from the System Advisor Model (SAM) database, were used. The PV module orientation was set to $180^{\circ}$ azimuth and $37^{\circ}$ tilt, resulting in an annual yield of $1135 \mathrm{kWh} / \mathrm{kWp}$ for 2014.Residential demand patterns were constructed from measurements in 400 dwellings with a 15 minute interval in 2008 and are provided by the authors of the Flex Street model [8]. Furthermore, electricity demand patterns of 26 medium to large commercial buildings were measured with a 15 minute interval in 2014. The PV yield profile and the demand profile were linear interpolated to a 5 minute interval for data alignment.

Battery charge and discharge was modelled with a battery algorithm for each timestep, shown in Eq. (3). The potential charge or discharge load is represented by $P_{\text {load pot. }}$ and the actual charge or discharge load by $P_{\text {load }}$. The potential energy charged to the battery or discharged from the battery is specified by $\Delta E_{\text {pot. }}$ and the definite energy by $\Delta E_{\mathrm{B}}$. The current battery state is given by $E_{\mathrm{B}}$ cur. and the next state by $E_{\mathrm{B} \text { next }}$. The minimum State Of Charge $(\mathrm{SOC})\left(\mathrm{SOC}_{\min }\right)$ value was set to $10 \%$ of the battery capacity, and the maximum SOC $\left(\mathrm{SOC}_{\max }\right)$ value to $90 \%$ of the battery capacity. The battery charge $\left(\eta_{\text {charge }}\right)$ and discharge $\left(\eta_{\text {discharge }}\right)$ efficiencies were set to $92 \%$. The maximum charge and discharge load $\left(P_{\max }\right)$ was set equal to the size of the PV system in $\mathrm{kWp}$.

$$
\begin{aligned}
& P=P_{\mathrm{PV}}-P_{\text {demand }} \\
& P_{\text {load pot. }}= \begin{cases}P \cdot \eta_{\text {charge }} & \text { if } P>0 \\
P \cdot \eta_{\text {discharge }} & \text { if } P<0 \\
0 & \text { if } P=0\end{cases} \\
& P_{\text {load }}= \begin{cases}P_{\text {load pot. }} & \text { if } P_{\text {load pot. }}<P_{\max } \\
P_{\max } & \text { if } P_{\text {load pot. }} \geq P_{\max }\end{cases} \\
& \Delta E_{\text {pot. }}=\sum_{t=t_{0}}^{t_{\text {end }}} P_{\text {load }}(t) \\
& E_{\mathrm{B} \text { pot. }}=E_{\mathrm{B} \text { cur. }}+\Delta E_{\text {pot. }} \\
& \Delta E_{\text {bat }} \begin{cases}\Delta E_{\text {pot. }} & \text { if } E_{\mathrm{B} \text { pot. }} \geq \mathrm{SOC}_{\mathrm{min}} \\
\Delta E_{\mathrm{pot}} & \text { if } E_{\mathrm{B} \text { pot. }} \leq \mathrm{SOC}_{\mathrm{max}} \\
E_{\mathrm{B} \text { cur }}-\mathrm{SOC}_{\text {min }} & \text { if } E_{\mathrm{B} \text { pot. }}<\mathrm{SOC}_{\text {min }} \\
\mathrm{SOC}_{\mathrm{max}}-E_{\mathrm{B} \text { cur }} & \text { if } E_{\mathrm{B} \text { pot. }}>\mathrm{SOC}_{\max }\end{cases} \\
& E_{\mathrm{B} \text { next }}=E_{\mathrm{B} \text { cur. }}+\Delta E_{\text {bat }}
\end{aligned}
$$

The annual demand of the residential and commercial patterns was scaled to the annual yield of a $1 \mathrm{kWp}$ PV system, thus comparing systems with differences in annual demand was possible. The influence of the battery size was evaluated using the Battery PV Ratio (BPVR). This is the ratio between the battery size in $\mathrm{kWh}$ and the PV system size in $\mathrm{kWp}$ (see Eq. (4)).

$$
\operatorname{BPVR}=\frac{\text { Battery size }[\mathrm{kWh}]}{\text { PV size }[\mathrm{kWp}]}
$$

\section{RESULTS}

\section{A. PV self-consumption}

The influence of the different BPVR values has been analysed for 400 residential and 26 commercial individual patterns. Fig. 1. shows the distribution of the self-consumption rate for the residential and commercial systems. The systems without batteries show an average SCR rate of 32\% for residential demand and $43 \%$ for commercial demand. The standard deviation for residential demands is $5 \%$ points whereas commercial demand shows $3 \%$ points. A higher standard deviation for residential systems is observed because of the larger range of energy usages in the residential patterns compared to the commercial patterns.

The distribution of a system with a BPVR of 1 shows a similar distribution shape as without batteries. The mean values of the distributions are increased towards $53 \%$ for residential and $62 \%$ for commercial systems. The standard deviation of systems with BPVR of 1 is similar as systems without battery. The average increase is larger for residential systems (21\% points) than for commercial systems (19\% points). Typically, in residential systems more electricity is stored to match the energy demand and PV supply than in commercial buildings. Consequently, battery energy storage results in a larger increase in self-consumption for residential systems.

Fig. 2 presents the boxplot distribution of the selfconsumption rate depending on the BPVR. A larger range of energy usages results in a greater boxplot range for the residential systems than commercial demand systems. This range of self-consumption rates is larger for residential demand than commercial demand for all analysed BPVR. However,

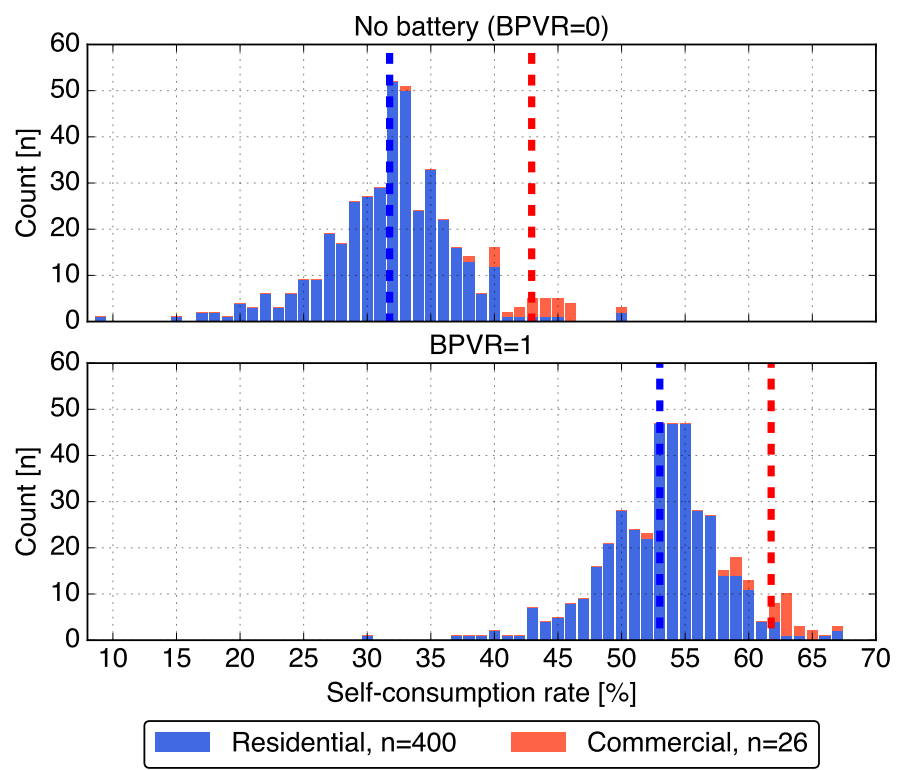

Fig. 1. Distribution of residential and commercial self-consumption rates for systems without battery (top) and systems with BPVR of 1 (bottom). Each bin represents 1\% SCR and the mean value of the distribution is indicated by the dotted lines. 

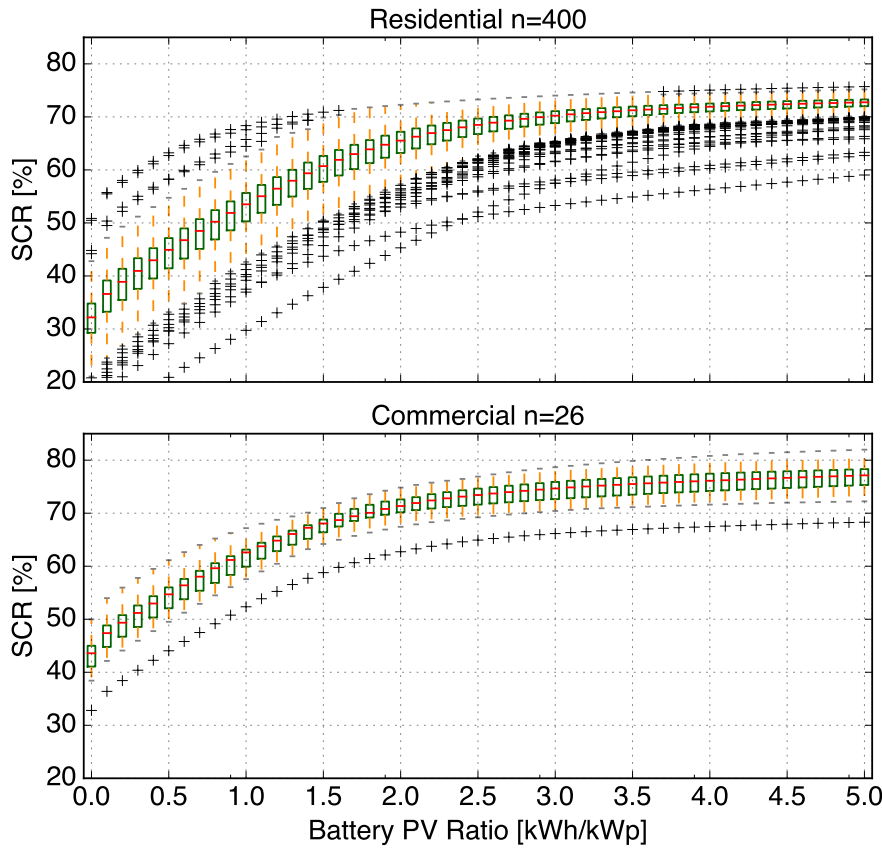

Fig. 2. Boxplot distribution of self-consumption rates for a residential systems (top) and commercial systems (bottom) dependent on BPVR. The plus signs indicate the outliers.

with the increase in BPVR, the range is decreasing for the residential patterns, but stays similar for the commercial patterns. Both boxplots distributions show two different behaviours dependent on the BPVR. The SCR is linear increased till $\mathrm{BPVR}<\sim 1.5$ for residential systems and $\mathrm{BPVR}<\sim 1$ for commercial systems. For higher values convergence occurs to a maximum of $\sim 70 \%$ for residential patterns and $\sim 75 \%$ for commercial patterns.

\section{B. Curtailment losses}

The curtailment losses are dependent on multiple factors, namely; feed-in limitation, PV system size, PV system yield pattern, demand pattern, demand size, battery size and battery algorithm systems. Fig. 3 shows the distribution of curtailment losses for residential and commercial systems without battery and with a battery size of BPVR $=1$. The commercial systems show a smaller range of curtailment losses compared to the residential systems because of the better match between the PV energy supply and the electricity demand. The mean value of the systems without battery is $7.7 \%$ for residential and $4.5 \%$ for commercial systems. The standard deviation for the residential systems is larger with $1.2 \%$ compared to commercial systems with $0.8 \%$. Adding a battery size of $\mathrm{BPVR}=1$ results in a larger decrease in curtailment losses for residential systems compared to commercial systems, specifically $0.9 \%$ point for residential and $0.5 \%$ point for commercial systems.

Fig. 4 presents the boxplot distribution of the systems for a range of BPVR values. The residential systems show a larger range compared to the commercial systems for all visible BPVR values. Both distributions show an $\mathrm{S}$ curve
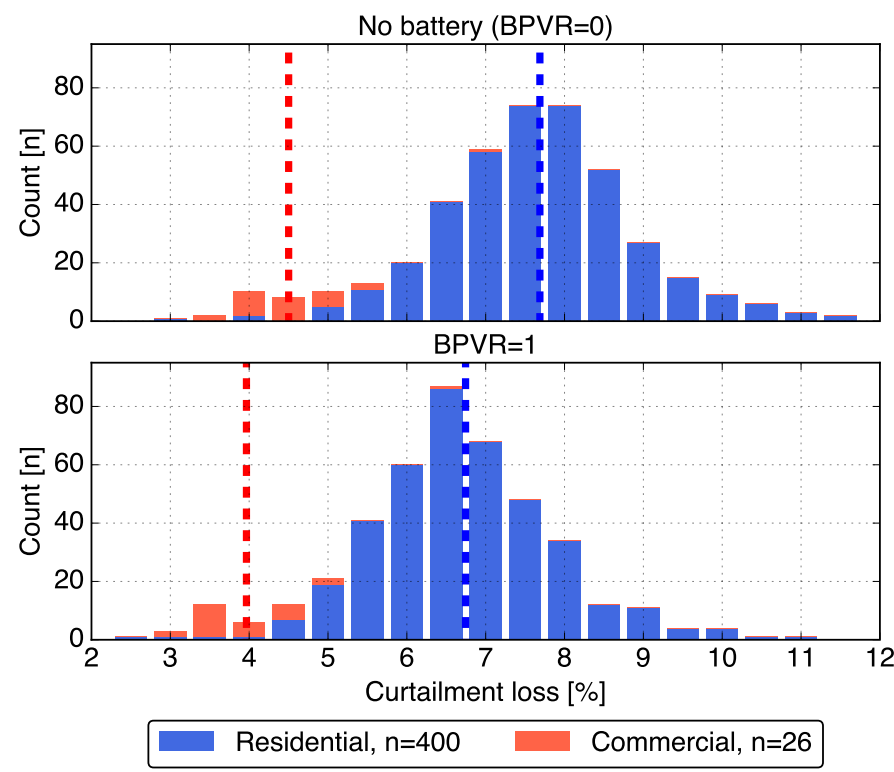

Fig. 3. Distribution of residential and commercial curtailment losses for a feed-in limitation of $0.5 \mathrm{~kW} / \mathrm{kWp}$ and two battery size options. Each bin represents $0.5 \%$ curtailment loss and the mean value of the distribution is indicated by the dotted lines.

behaviour indicating three different areas. Curtailment losses for $\mathrm{BPVR}<\sim 1$ show a small decrease, whereas the losses decrease faster between $B P V R=1$ and $B P V R=2$. The curtailment loss decreases slower for BPVR $>\sim 2$ and reaches $\sim 4 \%$ for residential systems and $\sim 2 \%$ for commercial systems.
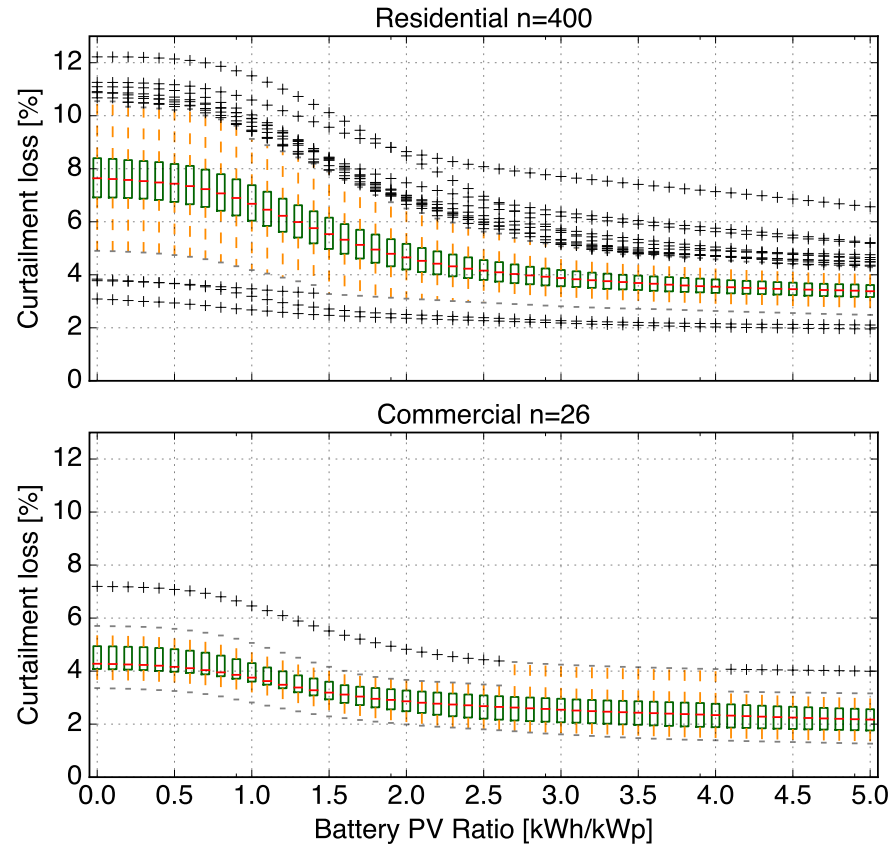

Fig. 4. Boxplot distribution of curtailment losses for a feed-in limitation of $0.5 \mathrm{~kW} / \mathrm{kWp}$ and dependent on BPVR. The plus signs indicate the outliers. 


\section{Discussion}

This study shows larger self-consumption rates for commercial systems than residential systems. The effect of batteries on the self-consumption rates shows similarity with previous studies [2], [3]. Differences can be explained by location, battery algorithm and demand patterns. The input data used are from measurements in the Netherlands and therefore results could differ for other locations. The curtailment losses presented are similar losses as in previous studies and adding an battery reduces these losses [3].

This study made several assumptions and has some limitations that could lead to different findings. Concerning the input data, it is neglected that the solar radiation influences the electricity demand patterns. The PV profiles were modelled with the measurement weather data from 2014, but the residential demand profiles are measured in 2008. Consequently, the impact of the solar radiation on the energy demand is not included. Also with the increasing electrification by heat pumps and electric vehicles the demand patterns will differ in the future. Second, The modelled PV yield is significantly higher than previous reported specific yield average of 875 $\mathrm{kWh} / \mathrm{kWp}$ for the Netherlands [9]. Only inverter losses are included in the modelled yield of $1135 \mathrm{kWh} / \mathrm{kWp}$, whereas for real PV systems additional losses occur, e.g. cable losses and shade losses. Also the orientation of the measured systems differs from the modelled systems. Furthermore, the self-consumption and curtailment losses were calculated using 5 minute interval data. Previous research shows that these parameters depending on the chosen time interval [6].

Regarding the method used, only a single simple battery algorithm and one feed-in limitation was investigated. If the battery algorithm would be optimized to reduce the feed-in towards the feed-in limitations, then the curtailment losses would be lower [3]. The impact of the battery on the curtailment losses would change with a different feed-in limitation. A limitation of $0.4 \mathrm{~kW} / \mathrm{kWp}$ would lead to a larger impact of the battery, whereas a limitation of $0.6 \mathrm{~kW} / \mathrm{kWp}$ would reduce the impact of the battery.

\section{CONCLUSION}

This study combined 400 residential and 26 commercial patterns with different PV system and battery sizes to analyse the self-consumption rate, self-sufficiency rate and curtailment losses. It was found that commercial systems show on average higher self-consumption rates and self-sufficiency rates, because better aligned of these demand patterns with the PV pattern. Batteries increase the self-consumption and self-sufficiency rates depending on the PV size and demand pattern. The residential demand patterns consist of a larger range of users, resulting in a larger range of SCR. Especially for this group, the individual demand patterns should be analysed to gain detailed knowledge of battery effect on the self-consumption. This is very useful for the economic profitability calculation of batteries.

The curtailment loss for a feed-in limitation of $0.5 \mathrm{~kW} / \mathrm{kWp}$ is on average larger for residential systems than for com- mercial systems. Also, a larger range of curtailment losses is seen for the residential systems. Furthermore, a battery results in decreased curtailment losses, however, this was not the main aim of the battery in this research. Especially the commercial systems show relative small losses of $<5 \%$ therefore using a battery only to reduce curtailment losses may not be economically viable yet.

Furthermore, it was found that the ideal battery size depends on the objective of the battery. A smaller battery has relatively more impact on the self-consumption rate, but a relative lower impact on the curtailment loss. A large battery has a relative smaller impact on the self-consumption rate, but a larger on the curtailment loss. To conclude, detailed economic analyses and the comparison of multiple battery algorithms is recommended for future work.

\section{ACKNOWLEDGMENT}

This work is part of the research programme Transitioning to a More Sustainable Energy System (grant number 022.004.023), which is financed by the Netherlands Organisation for Scientific Research (NWO). The authors are grateful to Hubert Spruijt, Machteld van den Broek and the Royal Dutch Meteorological Institute for providing data.

\section{REFERENCES}

[1] European Commission, "Best practices on Renewable Energy Selfconsumption,” European Commission, Brussels,, Tech. Rep., 2015.

[2] R. Luthander, J. Widn, D. Nilsson, and J. Palm, "Photovoltaic self-consumption in buildings: A review," Applied Energy, vol. 142 pp. $80-94,2015$. [Online]. Available: http://www.sciencedirect.com/ science/article/pii/S0306261914012859

[3] J. Weniger, T. Tjaden, and V. Quaschning, "Sizing of Residential \{PV\} Battery Systems," Energy Procedia, vol. 46, pp. 78-87, 2014. [Online]. Available: http://www.sciencedirect.com/science/article/ pii/S1876610214001763

[4] J. Li and M. A. Danzer, "Optimal charge control strategies for stationary photovoltaic battery systems," Journal of Power Sources, vol. 258, pp. 365 - 373, 2014. [Online]. Available: http://www.sciencedirect.com/science/article/pii/S0378775314002596

[5] A. Zeh and R. Witzmann, "Operational strategies for battery storage systems in low-voltage distribution grids to limit the feed-in power of roof-mounted solar power systems," Energy Procedia, vol. 46, pp. 114-123, 2014. [Online]. Available: http: //dx.doi.org/10.1016/j.egypro.2014.01.164

[6] J. Weniger, J. Bergner, D. Beier, M. Jakobi, T. Tjarko, and V. Quaschning, "Grid Feed-in Behavior of Distributed PV Battery Systems," in 31st European Photovoltaic Solar Energy Conference and Exhibition. WIPRenewable Energies, Munich, Germany, 2015, pp. 1726 - 1732.

[7] R. W. Andrews, J. S. Stein, C. Hansen, and D. Riley, "Introduction to the open source pv lib for python photovoltaic system modelling package," in 2014 IEEE 40th Photovoltaic Specialist Conference (PVSC), June 2014, pp. 0170-0174.

[8] F. N. Claessen, B. Claessens, M. P. F. Hommelberg, A. Molderink, V. Bakker, H. A. Toersche, and M. A. van den Broek, "Comparative analysis of tertiary control systems for smart grids using the Flex Street model," Renewable Energy, vol. 69, pp. 260-270, sep 2014. [Online]. Available: http://linkinghub.elsevier.com/retrieve/pii/ S0960148114002018

[9] W. G. van Sark, L. Bosselaar, P. Gerrissen, K. Esmeijer, P. Moraitis, M. van den Donker, and G. Emsbroek, "Update of the Dutch PV specific yield for determination of PV contribution to renewable energy production: 25\% more energy." WIP-Renewable Energies, Munich, Germany, 2014, pp. 4095 - 4097. 